\title{
Neue Strategieziele zur Behandlung erforderlich
}

\author{
Obwohl Inzidenz und Prävalenz der chronisch obstruktiven \\ Lungenerkrankung (COPD) zunehmen, gibt es kaum Studien, \\ die den Verlauf von der ersten schweren Exazerbation an \\ untersucht haben. Suissa et al. haben nun eine Studie \\ veröffentlicht, in der 73106 Patienten mit COPD über einen \\ Zeitraum von 17 Jahren (1990-2005) von der ersten schweren \\ Exazerbation an beobachtet wurden.
}

Thorax 2012; 67: 957-963

Als Ergebnis dieser Studie wurden 2 neue Strategieziele in der Behandlung der COPD definiert. Endpunkte waren COPDExazerbationen während des Krankenhausaufenthalts und Mortalität. Ausgeschlossen wurden Patienten unter 55 Jahren und Patienten, die zu einem früheren Zeitpunkt wegen eines Asthma bronchiale stationär behandelt worden waren. Das Durchschnittsalter der Pati- enten beim Aufnahme in die Studie lag bei 75 Jahren. Etwa 45\% der Studienteilnehmer (33166) erlitten mindestens eine schwere Exazerbation im Beobachtungszeitraum.

Der mittlere Abstand zwischen der 1. und der 2. Exazerbation betrug 5 Jahre und sank auf weniger als 4 Monate von der 9. zur 10. schweren Exazerbation. Es zeigte sich, dass nach der 2. Exazerbation das Risiko für ein erneutes Ereignis um das 3-Fache erhöht war, während es nach der 10. Exazerbation auf das 24-Fache anstieg. Die mittlere Überlebensrate lag bei 3,6 Jahren. Die Mortalität betrug 75\% nach 7,7 Jahren und 96\% nach 17 Jahren. In der ersten Woche nach stationärer Aufnahme lag die Mortalitätsrate nach einer schweren Exazerbation bei 40 pro 10000 pro Tag, nach 3 Monaten bei 5 pro 10000 pro Tag.

\section{Fazit}

Die rasche Verschlechterung des Gesundheitszustands nach der 2. Exazerbation und die hohe Mortalitätsrate in den Wochen nach jeder schweren Exazerbation erfordern neue Strategieziele, so die Autoren. Dies sei zum einen die Verzögerung einer 2. schweren Exazerbation und zum anderen das Verbessern der Behandlung von schweren Exazerbationen.

Kai Michael Schmidt-Borko, Polch

Krebszellen mit intaktem MALAT1. Ermutigt durch dieses Ergebnis prüften die Forscher, ob MALAT1 auch im intakten Organismus blockiert und damit die Metastasierung verhindert werden kann. Gemeinsam mit dem US-amerikanischen Unternehmen ISIS Pharmaceuticals entwickelten die Heidelberger Wissenschaftler kleine Nukleinsäure-Schnipsel (Antisense-Oligonukleotide), die von den Zellen aufgenommen werden und RNA-Moleküle gezielt blockieren. In Mäusen, denen menschliche Lungenkrebszellen injiziert wurden, verzögerten die MALAT1spezifischen Antisense-Schnipsel die Metastasenbildung: In den Tierlungen fanden sich weniger und kleinere Krebsherde als bei Artgenossen, die den Wirkstoff nicht erhalten hatten.

Dem Forscherteam gelang es erstmals, MALAT1 in Lungenkrebszellen in der Kulturschale nahezu vollständig auszuschalten. MALAT1, so entdeckten sie an den veränderten Zellen, reguliert zahlreiche Gene, die an der Metastasierung beteiligt sind. Das bewirkt u. a., dass die MALAT1negativen Tumorzellen in ihrer Beweglichkeit eingeschränkt sind und daher weniger invasiv in umgebendes Gewebe einwandern können. Wurden sie auf Mäuse übertragen, bildeten sie in der Lunge der Tiere deutlich weniger Tumorherde als
„Rund 10 Jahre nachdem wir MALAT1 als prognostischen Marker bei Lungenkrebs entdeckt haben, verstehen wir jetzt, wie diese nicht kodierende RNA die Metastasierung beeinflusst. Darüber hinaus hat sich die RNA als mögliches Zielmolekül für eine innovative Therapie mit AntisenseRNAs herausgestellt", so Diederichs Fazit.

Nach einer Mitteilung des Deutschen

Krebsforschungszentrums, Heidelberg Formen von Lungenkrebs als Marker fur MALAT1 die Tumorzellen bilden, desto wahrscheinlicher ist es, dass Metastasen der Studie, deren Ergebnisse Anfang 2013 im Fachjournal Cancer Research publiziert wurden, untersuchte sein Team auf welche gänge eingreift und dadurch die Metasta- die RNA MALAT1, die bei verschiedenen krankungen in Verbindung stehen. So auch oder den Zellt 